\title{
The significance of portal vein embolization in the treatment of colorectal liver metastases
}

\author{
P. ZBORIL ${ }^{1}$, K. VYSLOUZIL ${ }^{1, *}$, I. KLEMENTA ${ }^{1}$, P. SKALICKY ${ }^{1}$, K. VOMACKOVA ${ }^{1}$, M. CERNA ${ }^{2}$, K. CWIERTKA $^{3}$ \\ ${ }^{1}$ First Department of Surgery, Faculty of Medicine and Dentistry, Palacky University Olomouc; ${ }^{2}$ Department of Radiology, Faculty of Medicine \\ and Dentistry, Palacky University Olomouc; ${ }^{3}$ Department of Oncology, Faculty of Medicine and Dentistry, Palacky University Olomouc
}

*Correspondence: kamil.vyslouzil@fnol.cz

Received June 21, 2011 / Accepted October 3, 2011

\begin{abstract}
The first aim of the present paper was to evaluate hypertrophy of liver parenchyma after portal vein embolization in patients after systemic chemotherapy for colorectal carcinoma metastases and planned extensive liver resections. The second aim was to study whether hypertrophy of the liver parenchyma remnant after could influence the postoperative course large liver resections in long-term chemotherapy within complex therapy of colorectal carcinoma.

The prospective study comprised of 43 patients with colorectal hepatic metastases in whom liver resections of 4-5 segments were planned (Table 1). All patients underwent complex therapy of colorectal carcinoma, including chemotherapy consisting of 6-12 therapeutic cycles. Time interval between chemotherapy and liver resection was 2-24 months (mean interval of 8 months). Twenty patients whose presumed liver parenchyma remnant was less than $40 \%$ of total liver volume were indicated for portal vein embolization (mean liver parenchyma remnant of $29 \%$ ). This was always embolization of the right portal branch. Twenty-three patients were primarily indicated to liver resection.

Results: Hypertrophy of the left liver lobe occurred in all 20 patients. After portal vein embolization, the volume of left liver increased on average from $476 \mathrm{ml}(282-754)$ to $584 \mathrm{ml}(380-892)(\mathrm{P}<0.05)$. Mean hypertrophy of left liver lobe after portal vein embolization was $28.5 \%$. The measured parenchyma remnant after tumor resection increased from $29 \%$ up to $38 \%$ by hypertrophy. Mean values of ALT and AST in the postoperative period were significancantly different in the groups in this study. The values of alkaline phosphatase (ALP) and gamma glutamyl transpeptidase (GMT) were lower in patients after portal vein embolization $(\mathrm{P}<0.05)$. Significant differences were in postoperative level of serum bilirubin, bilirubin levels in patients after portal vein embolization were 2-3 times lower than in the group of patients after immediate surgery $(\mathrm{P}<0.05)$. The values of prothrombin time were also significantly lower in patients who underwent surgery without previous portal vein embolization $(\mathrm{P}<0.05)$.
\end{abstract}

Key words: colorectal carcinoma, liver metastases, chemotherapy, portal vein embolization

The mean survival of patients with hepatic metastases of colorectal cancer not subjected to conventional treatment is 6-9 months $(1,2)$. Radical surgery for liver metastases results in a 5 -year survival of over $30 \%$. However, the resectability of liver metastases is only about $30 \%(3,4,5,6)$. Preoperative portal vein embolization (PVE) was introduced into the treatment of nonresectable liver metastases with the aim of inducing involution of the involved liver lobe and to support compensation hypertrophy of the contralateral lobe (7). These changes may allow resection of the primarily nonresectable tumor or metastases in the liver and also minimize the risk of postoperative liver insufficiency after extended liver resection (8). Portal vein embolization is performed by introducing a direct probe into the portal vein branch under ultrasonography. Patients tolerate this intervention and the morbidity is very low. Portal vein embolization is indicated absolutely if the volume of the remnant liver parenchyma after liver resection is less than $25 \%$. If the volume of liver parenchyma residuum after liver resection amounts to $25 \%-40 \%$, the indication is relative and when after hepatectomy over $40 \%$ of liver parenchyma functions, portal vein embolization is not recommended. In exceptional cases, several authors have advocated portal vein embolization for interventions when $45 \%$ of liver parenchyma remains fully functional after resection $(9,10,11)$. However, hypertrophy of the parenchyma of the contralateral lobe one month after 
embolization is variable. The volume of the contralateral lobe may increase by $15 \%$ to $90 \%$. (8).

The introduction of portal vein embolization into the therapy of primarily nonresectable liver metastases has resulted in a significantly greater number of patients with liver cancer who are subjected to radical surgery. According to the study published by Azulay et al. at the Hospital Paul Brouse Villejuif (12), this method has extended the resectability of liver metastases and carcinomas by $19 \%$. In addition to extended resectability, a more favorable postoperative course was found in patients undergoing portal vein embolization. Hypertrophy of the contralateral lobe may positively influence the postoperative function of the remaining liver parenchyma and prevent postoperative liver failure. The present study is focused on the possible effects of portal vein embolization on the postoperative dynamics of liver function and the postoperative course of patients, who had undergone adjuvant chemotherapy. The aim of this study was not to evaluate overall and disease free survival.

\section{Methods and patients}

In the course of a five-year period (2002-2008) we treated 48 patients indicated for extensive liver resections for colorectal liver metastases. The prospective study comprised 43 patients who underwent liver resections within 4-5 segments. Clinicopathological characterization of the patient cohort is shown in Table 1. Five patients were excluded: two patients

Table1. Group of 43 patients with extensive liver resection for colorectal carcinoma metastases in between 2002-2006 (Fisher's exact test did not show a significant difference between the group of patients with embolisation and without embolisation in gender $(p=1.000)$, in type of metastasis $(p=1.000)$, in the number of metastases $(p=1.000))$.

\begin{tabular}{l|c|c}
\hline & $\begin{array}{c}\text { Preoperative PVE } \\
(\mathrm{n}=20)\end{array}$ & $\begin{array}{c}\text { Without preoperative PVE } \\
(\mathrm{n}=23)\end{array}$ \\
\hline Men/women & $12 / 8$ & $13 / 10$ \\
Mean age (years) & 62,3 & 65,2 \\
Type of metastases & & \\
$\quad$ Synchronous & 12 & 14 \\
$\quad$ Metachronous & 8 & 9 \\
No. of metastases & & \\
$\quad$ 1-3 & 13 & 15 \\
$\quad$ over 3 & 7 & 8 \\
Type of liver resection & 14 & 15 \\
RH & 4 & - \\
RH+S4 & 1 & - \\
RH+S1 & - & 1 \\
RH+S2 & 1 & - \\
RH + S3 & - & 2 \\
LH +S6+S8 & - & 2 \\
LH+S5 & - & 3 \\
LH + S6 & & \\
\hline
\end{tabular}

Explanation: PVE - portal vein embolization

$\mathrm{RH}$ - right hepatectomy

LH - left hepatectomy

S - segmentectomy with higher operative risk than the ASA II score, one patient with chronic liver disease and two patients with extrahepatic malignant dissemination. Twenty-four patients exhibited primary adenocarcinoma of the rectum, seven patients primary adenocarcinoma of the left colon and twelve patients primary adenocarcinoma of the right colon. All included patients were subjected to radical surgery of the colorectal carcinoma with adjuvant chemotherapy comprising 6-12 therapeutic cycles. The time interval between completed chemotherapy and liver resection was 2-24 months (mean 8 months). Twenty patients in whom the remaining liver parenchyma did not exceed $40 \%$ of total liver volume according to preoperative CT were indicated for right portal branch embolization (mean remaining liver parenchyma was $29 \%$ ). The volume of liver parenchyma was measured in 3D CT reconstruction and tissue volume was calculated in ml. Twenty-three patients in whom CT showed more than $40 \%$ of remaining liver parenchyma were indicated for primary liver resection.

Portal vein embolization was performed according to procedure described by Makuuchi (13). All patients were subjected to CT measurement of the left liver parenchyma remnant prior to surgery. Those indicated for portal vein embolization were also subjected to CT assessment of the volume of right and left liver lobes before portal vein embolization. We thus assessed the volume of the left parenchyma residuum after planned liver resection in patients indicated for portal vein embolization and the extent of left parenchyma hypertrophy caused by embolization. Patients underwent surgery 6-8 weeks after portal vein embolization.

All liver resections were performed by the same team. During lobectomy, vascular and biliary structures were dissected in the porta hepatis for the appropriate lobe, and then ligated, and liver parenchyma was resected. In the case of extra-anatomical resections, liver parenchyma resection was performed during temporary clamping of hepatic pedicle and a harmonic scalpel was used. Immediately after the surgery, the patients were given artificial ventilation; extubation was carried out 6-8 hours after the surgery. Postoperative parenteral hepatoprotective feeding with gradual alimentation was identical for all patients. Parenteral feeding lasted for 5 days. No complications requiring immediate operational revision occurred.

The data were analysed using statistical software SPSS version 15 (SPSS Inc., Chicago, USA). The categorical variables were compared using Fisher's exact test. The Mann-Whitney $\mathrm{U}$-test was used to compare continuous variables.

Pair data were analysed using Wilcoxon signed-rank test with Bonferroni correction of significance. The normality of distribution was checked by the Shapiro-Wilk test. The results were considered statistically significant $(\mathrm{P}<0.05)$.

\section{Results}

Portal vein embolization was successful in all 20 patients. All showed a transient increase in liver function tests after the procedure. Eighteen patients were discharged within 5 


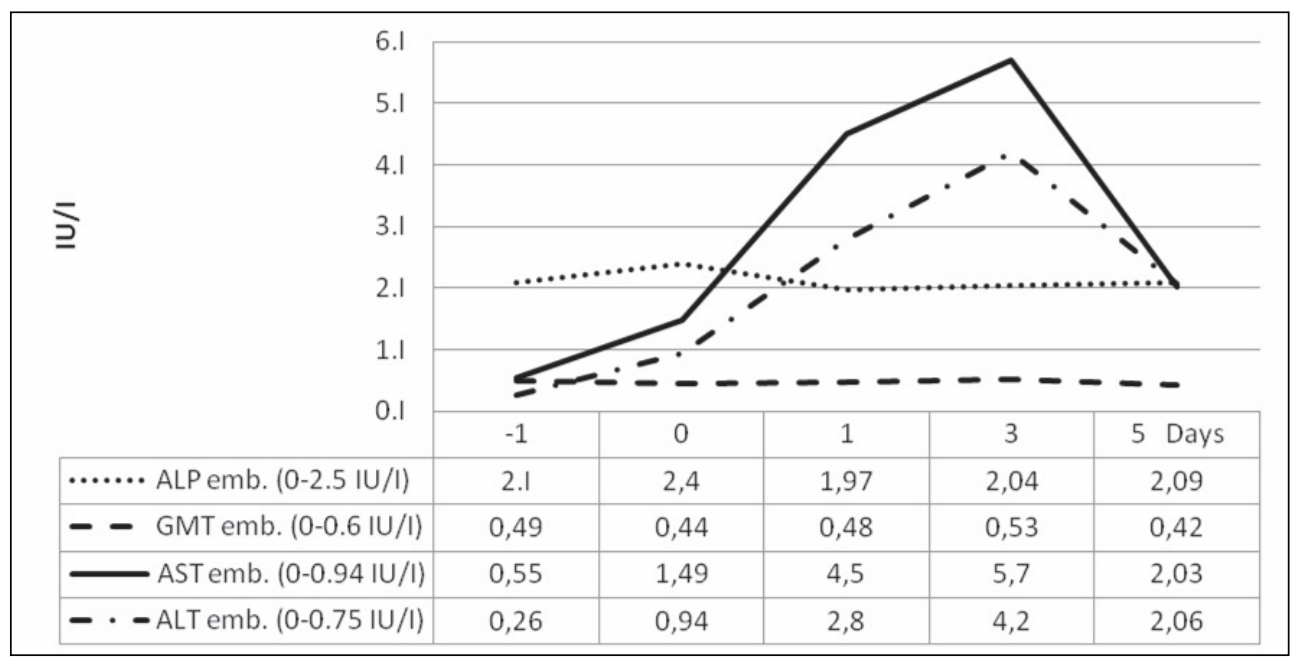

Fig. 1. Values of liver tests (ALP, GMT, AST, ALT) after PVE $(0=$ day of PVE).

Comparison -1 st day vs. 0, 1st, 3rd and 5th day after embolisation (results of Wilcox paired test with Bonferroni correction of signifance)

For parameters AST and ALT, a significant elevation was seen on the $\mathbf{0 , 1}$ st, and 3rd days after embolisation in comparison to the values on the day before the embolisation $(p<0.001)$, on the 5 th day after the embolisation a significant decrease was observed compared with the 1st and 3 rd days after embolisation, the values, however, remained significantly higher compared to the values prior to the embolisation $(\mathbf{p}=0.0004)$.

No significant change was observed for ALP and GMT values after embolisation.

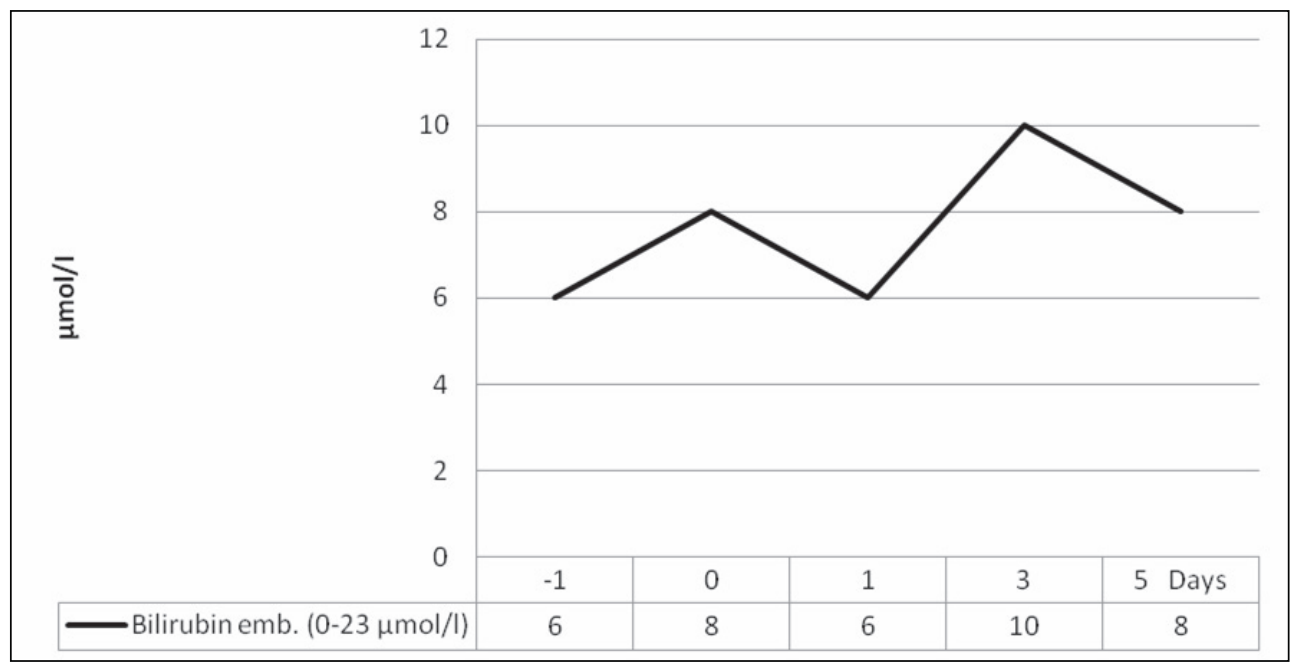

Fig. 2. Values of bilirubin after PVE $(0=$ day of PVE $)$.

Comparison -1 st day vs. 0, 1st, 3rd and 5th day after embolisation (results Wilcox paired test with Bonferroni correction of significance)

The values of bilirubin on the 0 and 3rd day were significantly higher compared to the values on the day before the embolisation $(p=0.018, p=0.001$ respectively). On the 5th day after embolisation decrease was observed compared to the 3 rd day after embolisation, the values, however, remained significantly higher in comparison with the values before embolisation $(p=0.043)$. The values of bilirubin on the 1 st day after embolisation did not differ significantly from the values on the day before embolisation $(p=1.000)$.

days of embolization. Mean values of liver tests after portal vein embolization of these patients are given in figures 1-2. Asparate-amino transferase (AST) and alanine-amino transferase (ALT) increased with a maximum on the $3^{\text {rd }}$ day after embolization, then laboratory values returned to normal within 10 days. Two patients with 12 cycles of chemotherapy, completed 2 and 3 months respectively before embolization, showed a significant increase in liver tests persisting for 14 days. These patients had in addition to high AST and ALT increased serum bilirubin and alkaline phosphatase (AP). Laboratory values for patients subjected to embolization returned to normal 6-8 weeks after embolization and also in 


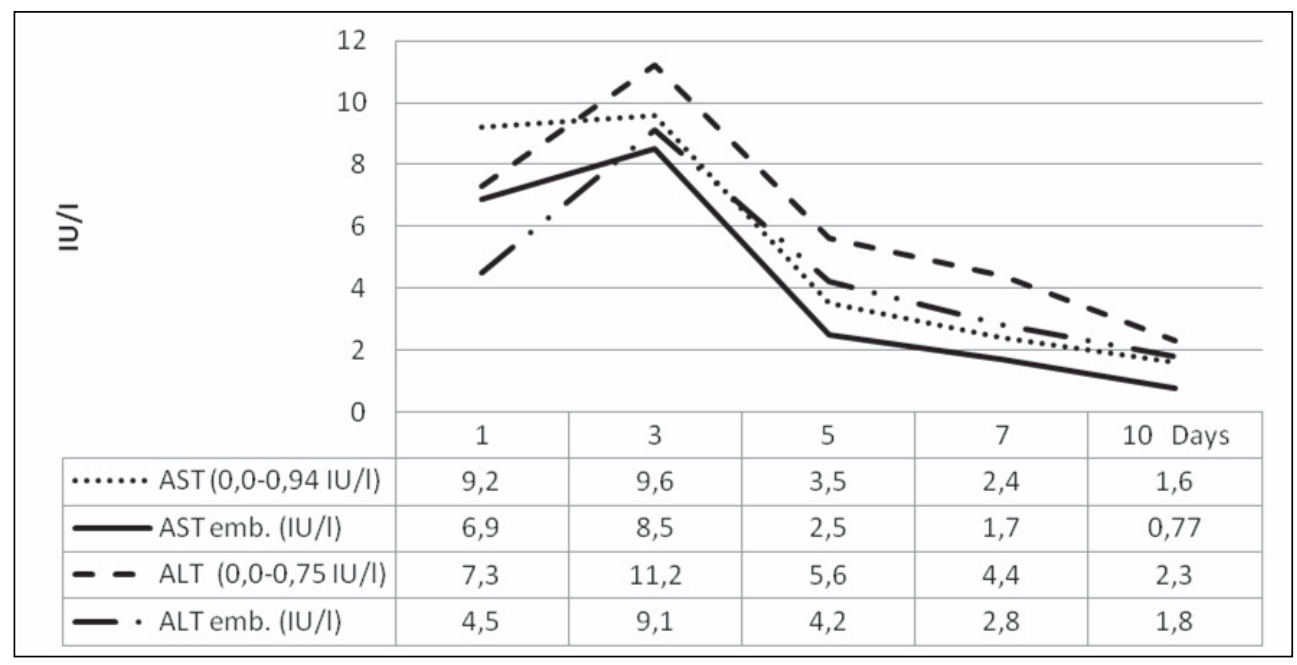

Fig. 3. Values of liver tests (AST, ALT) after liver resection.

the laboratory test before liver resection, the laboratory values were normal in all patients.

Hypertrophy of the left liver occurred in all 20 patients. Six weeks after portal vein embolization, the volume of the left liver lobe increased from $476 \mathrm{ml} \mathrm{(282-754)} \mathrm{to} 584 \mathrm{ml} \mathrm{(380-892)} \mathrm{(P}$ $<0.05)$. The mean increase in left liver lobe after portal vein embolization was $28.5 \%$.

When comparing the parenchyma remnant after presumed liver resection, portal vein embolization induced a mean increase in liver parenchyma remnant from $29 \%$ to $38 \%$. On the basis of measurement, all patients were able to undergo liver resection with sufficient functional reserve of the liver parenchyma residuum.

The extent of liver resections is shown in Table 1. In the group subjected to surgery after portal embolization, 4 patients (20\%) developed postoperative complications, two cases of postoperative ascites and two cases of pulmonary inflammatory infiltration. Of 23 patients who underwent surgery without preoperative portal vein embolization, 8 patients (35\%) experienced postoperative complications (Table 2). One patient suffered a fatal liver insufficiency. Two patients expe-

Table 2. Postoperative complications. The group of patients with embolisation and without embolisation did not differ significantly in the number of postoperative complications ( $20 \%$ vs. $35 \%, p=0.327$, Fisher's exact test).

\begin{tabular}{l|c|c}
\hline & $\begin{array}{c}20 \text { patients after } \\
\text { PVE }\end{array}$ & $\begin{array}{c}23 \text { patients without } \\
\text { PVE }\end{array}$ \\
\hline Liver failure & 0 & 1 \\
Ascites & 2 & 2 \\
Pulmonary complications & 2 & 2 \\
Biliary fistula & 0 & 1 \\
Perihepatic bile collection & 0 & 2 \\
Total complications & 4 & 8 \\
\hline
\end{tabular}

rienced transient ascites, one patient developed a peripheral biliary fistula which healed spontaneously, and two patients developed perihepatic bile collection which was managed by probe under CT with transient pigtail drainage. Two cases of bronchopneumonia required antibiotic therapy with rehabilitation and bronchoscopic aspiration in one patient.

Evaluation of the postoperative function of liver parenchyma remnant comprised evaluation of serum bilirubin, AST, ALT, gamma glutamyl transpeptidase (GMT), alkaline phosphatase and prothrombin time in the course of 10 postoperative days. The kinetics of postoperative liver tests and prothrombin time are summarized in Figs 3-7. Statistical analysis showed that significant difference in AST levels between the group of patients with embolisation and without was seen only on the first postoperative day, median AST values were 6.9 for embolisation patients vs. 8.9 for patients without embolisation ( $\mathrm{p}=0.031$, Mann-Whitney test).

In the group of patients with embolisation, significantly lower values of ALT were observed on the first, fifth, seventh and tenth postoperative days in comparison to the group without embolisation $(\mathrm{p}=0.003, \mathrm{p}=0.047, \mathrm{p}=0.005, \mathrm{p}=$ 0.024 , Mann-Whitney test). The third postoperative day did not show a significant difference $(\mathrm{p}=0.082)$.

Levels of alkaline phosphatase in the group with embolisation showed significantly lower values on the third, fifth, seventh and tenth postoperative days in comparison to the group without embolisation $(p=0.012, p=0.036, p=0.0002$, $\mathrm{p}=0.033$, Mann-Whitney test). The first postoperative day did not show significant difference $(\mathrm{p}=0.884)$.

Similarly in the group with embolisation, significantly lower GMT values were seen on the third, fifth and seventh postoperative days in comparison to the group without embolisation ( $\mathrm{p}=0.017, \mathrm{p}=0.0005$ and $\mathrm{p}=0.001$, Mann-Whitney test). The first and tenth postoperative days did not show significant difference. 


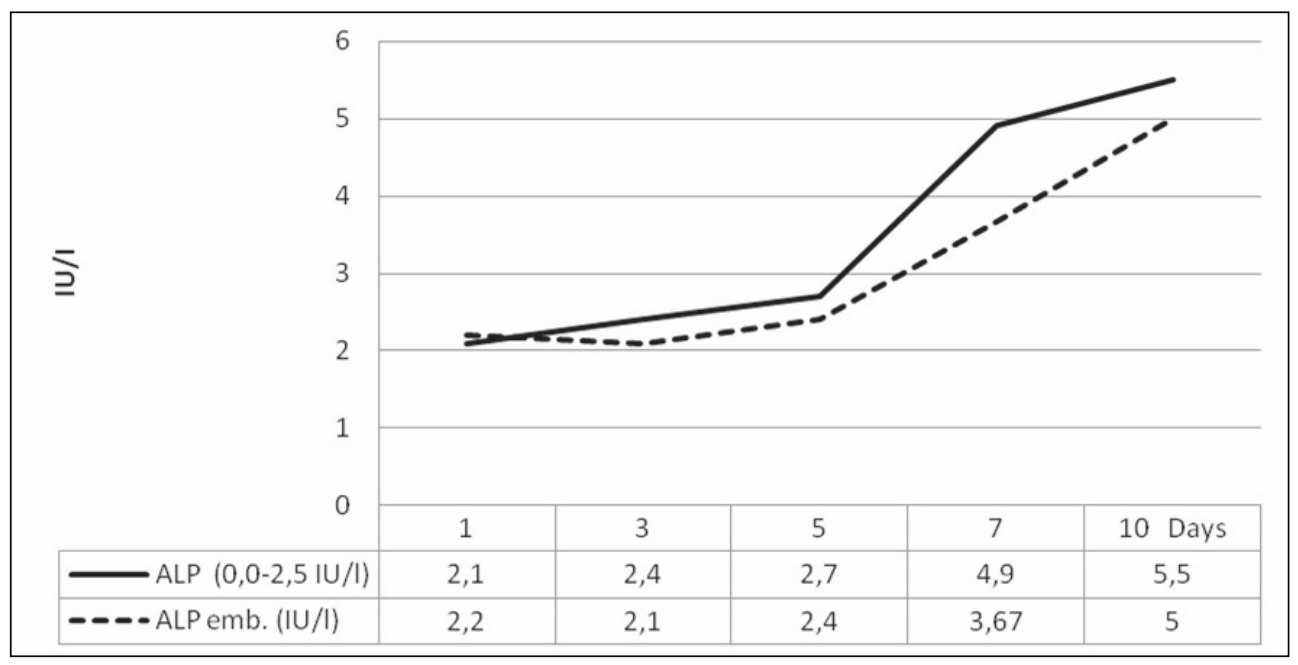

Fig. 4. Values of liver tests (ALP) after liver resection.

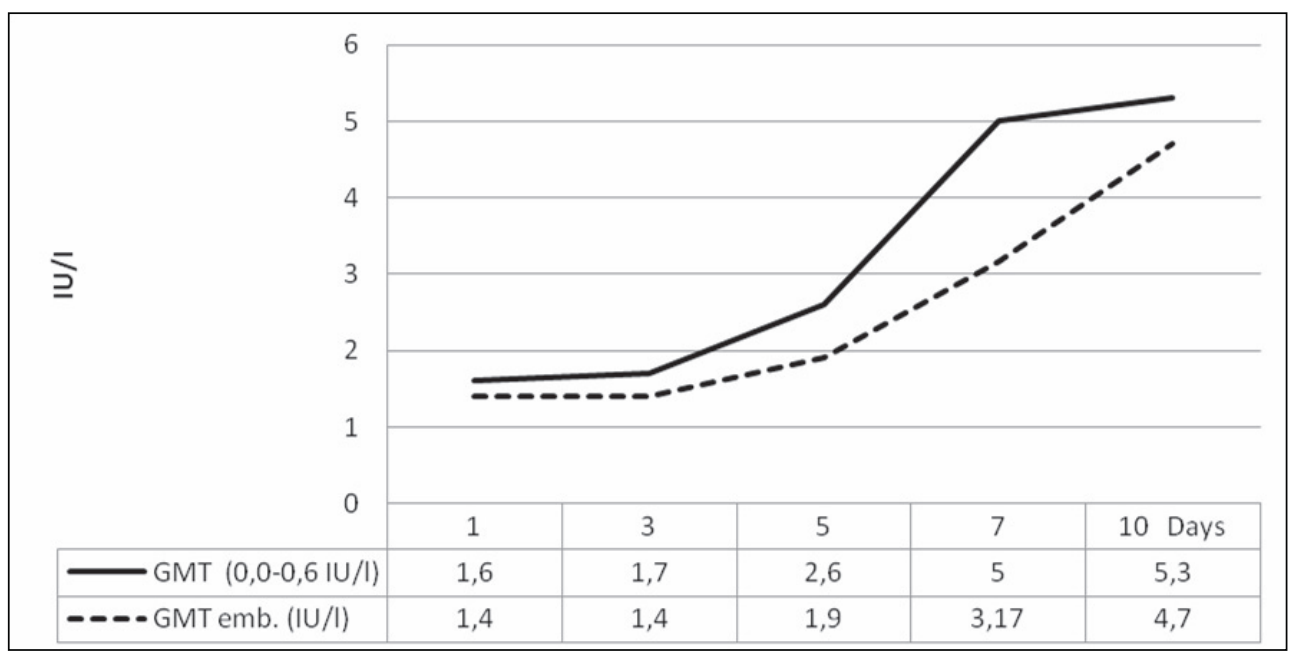

Fig. 5. Values of liver tests (GMT) after liver resection.

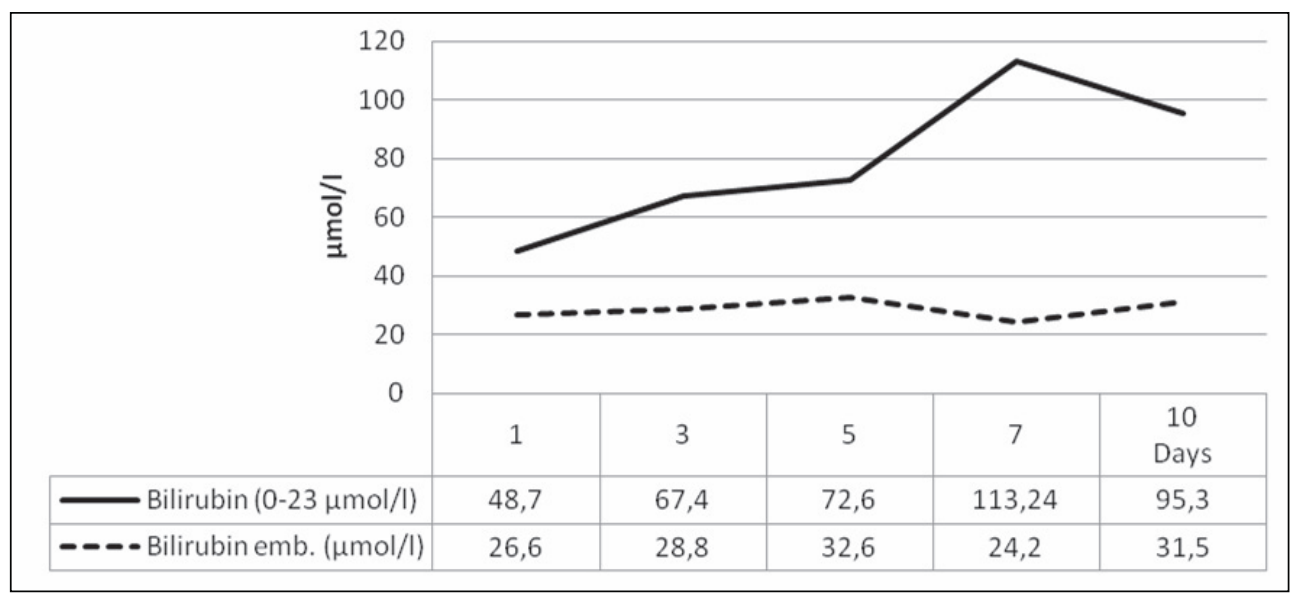

Fig. 6. Values of bilirubin after liver resection. In the group of patients with embolisation, significantly lower values of bilirubin were seen on all postoperative days compared with the group without embolisation $(p<0.0001$ for all days, Mann-Whitney test). 


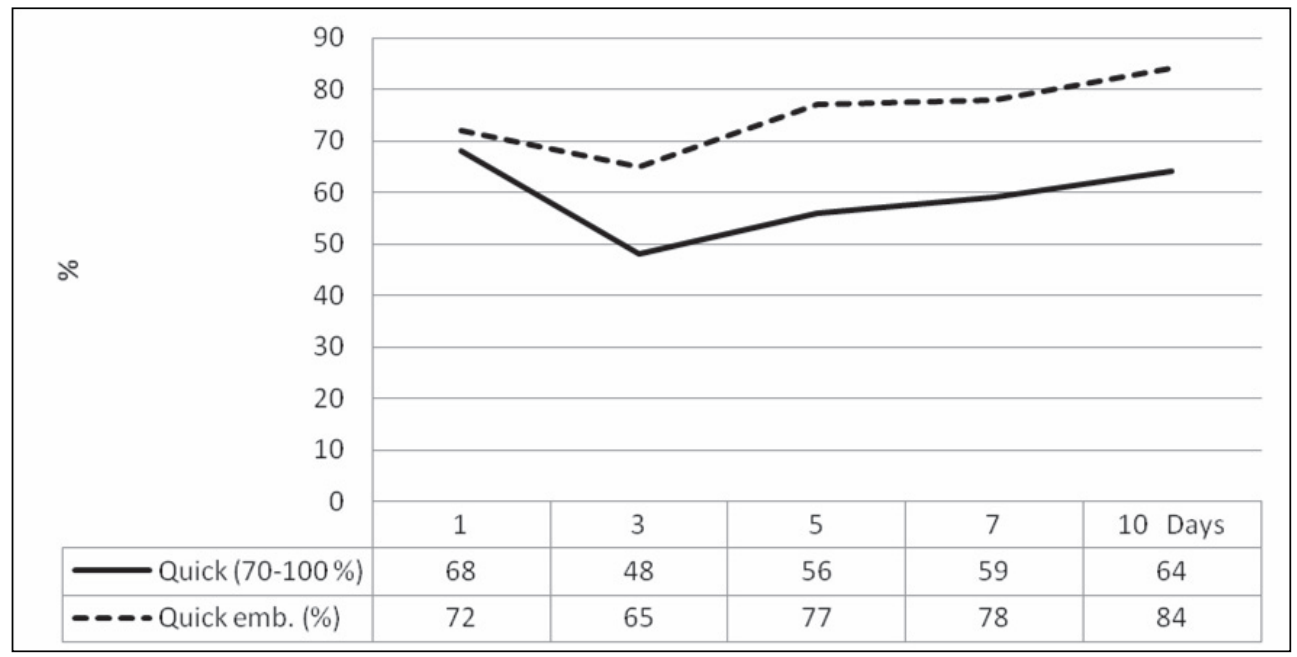

Fig. 7. Values of prothrombin time after liver resection in both groups of patients. In the group of patients with embolisation, significantly higher values of prothrombin time were observed on the $3 \mathrm{rd}, 5 \mathrm{th}, 7$ th and 10th postoperative days in comparison with the group without embolisation ( $\mathrm{p}<0.0001$, Mann-Whitney test). On the 1st postoperative day no significant difference was observed $(p=0.217)$.

Significant differences were found in postoperative serum bilirubin where bilirubin levels in patients after portal vein embolisation were 2-3 times lower than in patients subjected to immediate surgery $(\mathrm{P}<0.05)$ (Fig. 6). The prothrombin time was also significantly lower in patients without preoperative portal vein embolization $(\mathrm{P}<0.05)$ (Fig.7).

The mean hospital stay of patients subjected to liver resection after portal vein embolisation was 15 days (range 11-28) and of patients without portal vein embolisation 17 days (range 10-35) $(\mathrm{P}=\mathrm{NS})$.

\section{Discussion}

Extensive liver resections for colorectal carcinoma metastases have been performed more frequently in patients after long-term adjuvant or neoadjuvant chemotherapy. The present study focused not as much on the extent of left liver hypertrophy after preoperative portal vein embolization as on the postoperative kinetics of liver function in these patients. Multiple studies emphasise the importance of portal vein embolization in patients in whom the primary liver resection cannot be performed due to large tumor involvement of liver. Other studies describe the beneficial postoperative course after portal vein embolization in patients with chronic liver disease $(7,10,12,14,15,16,17)$. In our group the aim of study was focused on patients with assumed chronic effects of chemotherapy on liver parenchyma function. Our results indicate that patients with long-term chemotherapy should be considered as patients with chronic hepatopathy. After portal vein embolization, the left liver enlarged on average by $28.5 \%$ after 6 weeks. The volume of liver parenchyma remnant increased from $29 \%$ to $38 \%$. These results correspond to studies dealing with the effect of portal vein embolization on patients with chronic liver disease $(10,18,19)$. The follow-up of postoperative liver tests showed the beneficial effect of portal vein embolization on the function of liver cells in patients after long-term chemotherapy. Although preoperative portal vein embolization was indicated in patients whose post-resection liver parenchyma remnant was probably less than $40 \%$, this group had significantly better postoperative dynamics of liver function (patients without portal vein embolization = liver parenchyma remnant over $40 \%$ ). Statistically significant differences in the kinetics of ALP, GMT and mainly bilirubin and prothrombin time indicated the beneficial contribution of preoperative portal vein embolization in patients even after long-term chemotherapy and planned extensive liver resection for colorectal carcinoma metastases.

After portal vein embolization, signs of obstructive icterus may appear on the basis of cholestasis. Koyama et al. (20) found out that cholestasis reduces the metabolic function of liver cells, including their mitochondrial function. A cholestatic liver was found to have an increased volume of only $7.2 \%$ compared to $25.7 \%$ in non-cholestatic parenchyma. In our cohort of 20 patients after PVE, two showed signs of cholestasis.These patients had undergone 12 cycles of chemotherapy, which were completed 2 and 3 months respectively prior to PVE. Hypertrophy of the left liver lobe was present in 19\% and $22 \%$ respectively. Thus it may be stated that cholestasis after PVE had minimal effect on hypertrophy of the left liver lobe. In the remaining 18 patients a transitory elevation of ALT and AST was observed. Due to the fact that the patients were after long-term chemotherapy, the elevated ALT and AST cannot be evaluated as solely a result of PVE, but the effect of chemotherapy on liver cells must be considered. Our results show that changes in liver function following PVE do not correlate with changes in liver function after extensive liver resection. 
Indications for portal vein embolization are not specified only by extent of liver parenchyma resection and/or quantitative evaluation of liver parenchyma residuum after resection, but also on functionality of the liver parenchyma remnant. It is not easy to determine whether the remaining liver parenchyma will function sufficiently after extensive liver resection without liver failure. Methods for imaging the volume of liver parenchyma (CT with $3 \mathrm{D}$ reconstruction) show the probable volume of liver parenchyma residuum after resection, but they cannot indicate anything more about postoperative liver function. Functional tests based on the follow-up of liver metabolism determine the preoperative function of liver parenchyma as a whole, but are insufficient to determine reliably the quality of liver parenchyma remnant (10). Post-resection insufficiency of liver parenchyma may manifest early after surgery as coagulopathy, with subsequent postoperative complications and unfavorable prognosis.Another manifestation of liver parenchyma insufficiency is icterus with high values of mostly conjugated bilirubin. These states of chronic liver insufficiency may be lethal as well $(21,22)$. During preoperative assessment of the extent of surgical intervention, comorbidities should be taken into consideration. Important are diseases affecting the stability of arterial/venous blood pressure, or diminished oxygenation. In these patients, postoperative ventilation should be applied with monitoring of liver functions (23, $24,25,26,27,28,29$ ).

Based on the results of the present study, preoperative portal vein embolization should be considered beneficial for patients in whom extensive liver resections are planned for metastases after long-term chemotherapy.

Conclusions. Preoperative portal vein embolization may both increase the number of patients eligible for liver resection and increase the safety of resection in patients on long-term chemotherapy. Statistically significant differences in the kinetics of ALP and GMT but mainly bilirubin and prothrombin time indicate the benefits of preoperative portal vein embolization in these patients and planned extensive liver resection for colorectal carcinoma metastases.

\section{References}

[1] JAECK D, BACHELIER P, GUIGUET M, et al. Long-term survival following resection of colorectal hepatic metastase. Br J Surg 1997; 84: 977-980. http://dx.doi.org/10.1002/ bjs. 1800840719

[2] SKIBBER JM, MINSKY BD, HOFF PM. Cancer of the Colon, Cancer of the rectum. In Principles and Practice of Oncology. Philadelphia: Lippincott; 2001.

[3] HUGH T, POSTON G. The aetiology and management of hepatic metastases. Aust NZ J Surg 1997; 67: 400-409. http:// dx.doi.org/10.1111/j.1445-2197.1997.tb02003.x

[4] LIU LX, ZHANG WH, JIANG HC. Current treatment for liver metastases from colorectal cancer. World J Gastroenterol 2003; 9: 193-200.
[5] PENNA C, NORDLINGER B. Surgery of liver metastases from colorectal cancer: new promises. Br Med Bull 2002; 64: 127-40. http://dx.doi.org/10.1093/bmb/64.1.127

[6] RUERS T, BLEICHRODT RP. Treatment of liver metastases, an update on the possibilities and results. Eur J Cancer 2002; 38: 1023-1033. http://dx.doi.org/10.1016/S0959-8049(02)00059$\underline{\mathrm{X}}$

[7] ABDALA EK, HICKS ME, VAUTHEY JN. Portal vein embolization: rationale, technique and future prospects. $\mathrm{Br}$ J Surg 2001; 88: 165-175. http://dx.doi.org/10.1046/j.13652168.2001.01658.x

[8] ADAM R. Chemotherapy and surgery: new perspectives on the treatment of unresectable liver metastases. Ann Oncol 2003;14 (suppl.2): 13-16. http://dx.doi.org/10.1093/annonc/ mdg731

[9] IMAMURA H, SHIMADA R, KUBOTA S, et al. Preoperative portal vein embolization: an audit of 84 patients. Hepatology 1999; 29: 1099-1105. http://dx.doi.org/10.1002/ hep. 510290415

[10] UESAKA K, NIMURA Y, NAGINO M. Changes in hepatic lobar function after right portal vein embolization. Ann Surg 1996; 223: 77-78. http://dx.doi.org/10.1097/00000658199601000-00011

[11] URATA K, HASHIKURA Y, OMEGAMI T, et al. Standard liver volume in adults. Transplant Proc 2000; 32: 2093-2094. http://dx.doi.org/10.1016/S0041-1345(00)01583-9

[12] AZULAY D, CASTAING D, SMAIL A, et al. Resection of nonresectable liver metastases from colorectal cancor after percutaneous portal vein embolization. Ann Surg 2000; 231: 480-86. http://dx.doi.org/10.1097/00000658-200004000$\underline{00005}$

[13] MAKUUCHI M, THAI BL, TKAYASU K, et al. Preoperative portal embolization to increase safety of major hepatectomy for hilar bile duct carcinoma: a preliminary report. Surgery 1990; 107: 521-527

[14] ELIAS D, DEBAERE T, ROCHE A, et al. Preoperative selective portal vein embolizations are an effective means of extending the indications of major hepatectomy in the normal and injured liver. Hepato-Gastroenterology 1998; 45: 170-177

[15] ELIAS D, OULLET JF, DE BAERE T. Preoperative selective portal vein embolization before hepatectomy for liver metastases: long-term results and impact on survival. Surgery 2002; 131: 294-299. http://dx.doi.org/10.1067/msy.2002.120234

[16] TANAKA H, HIROHASHI K, KUBO S, et al. Preoperative portal vein embolization improves prognosis after right hepatectomy for hepatocellular carcinoma in patients with impaired hepatic function. Br J Surg 2000; 87: 879-882. http:// dx.doi.org/10.1046/j.1365-2168.2000.01438.x

[17] VAUTHEY JN, CHAOUI A, DO KA, et al. Standardized measurement of the future liver remmnant prior to extended liver resection: methodology and clinical associations. Surgery 2000; 127: 512-519. http://dx.doi.org/10.1067/msy.2000.105294

[18] ADAM R. Facteurs pronostiques des hepatectomies apres chimiotherapie. JHB 2002; 11:30.

[19] FRIEDBERG B, WATINE J, MIEDOUGE M. Unresected colorectal liver metastases: prognostic value of laboratory variables. Gastroenterol Clin Biol 2001; 10: 962-966. 
[20] KOYAMA K, TAKAGI Y, ITO K. Experimental and clinical studies on the effect of billiary drainage in obstructivee jaudice. Am J Surg 1981; 142: 293-299. http://dx.doi.org/10.1016/00029610(81)90296-8

[21] WAKABAYASHI H, ISHIMURA K, OKANO K, et al. Application of preoperative portal vein embolization before major hepatic resection in patients with normal or abnormal liver parenchyma. Surgery 2002; 131:26-33. http://dx.doi. org/10.1067/msy.2002.118259

[22] ZIMMERMANN H, REICHEN J. Hepatectomy: preoperative analysis of hepatic function and postoperative liver function. Dig Surg 1998; 15: 1-11. http://dx.doi.org/10.1159/ 000018578

[23] ALEXANDER HR. Surgical approaches to liver metastases. Cancer 2002; 8: 68-81.

[24] DOCI R, GENNARI L, BIGNAMI P, et al. One hundred patients with hepatic metastases from colorectal cancer treated by resection: analysis of prognostic determinants. Br J Surg 1991; 78: 797-801. http://dx.doi.org/10.1002/bjs.1800780711

[25] FIORENTINI G, PODDIE DB, CANTORE M. Locoregional therapy for liver metastases from colorectal cancer: the possibilities of intraarterial chemotherapy, and new hepatic-directed modalities. Hepato-Gastroenterology 2001; 48: 305-312 .

[26] FISHMAN AD, WADLER S. Advances in treatment of metastatic colorectal cancer. Clin Colorectal Cancer 2001; 1: 20-35. http://dx.doi.org/10.3816/CCC.2001.n.002

[27] OTT R, WEIN A, HOHENBERGER W. Liver metastasesprimary or multimodal therapy? Chirug 2001; 72: 887-897. http://dx.doi.org/10.1007/s001040170084

[28] POSTON G. The argument for liver resection in colorectal liver metastases. Hepato-Gastroenterology 2001; 48: 345-346.

[29] RAVIKUMAR TS, GALLOS G. Resection of the liver metastases: state of the art. Oncology 2002; 16: 1240-1256. 